\title{
Über Nickelsuperoxyd, besonders sein Verhalten bei der Salzbildung.
}

\author{
Von \\ C. Tubandt und W. Riedei.
}

Seitdem es Bellucci und Cravari ${ }^{1}$ gelungen ist, ein Nickeldioxyd darzustellen und einwandfrei nachzuweisen, dals dasselbe bei allen Oxydationen von Nickeloxydulverbindungen gebildet wird, ist man vielfach geneigt, mit den genannten Autoren anzunehmen, dals dieses Dioxyd das einzige, wirklich existierende höhere Oxyd des Nickels darstellt, das sonst wohl charakterisierte ${ }^{2}$ Nickelsesquioxyd also nicht als besondere Oxydationsstufe des Nickels, sondern wie auch die anderen noch bekannten höheren Nickeloxyde als Verbindung von $\mathrm{NiO}_{2}$ und $\mathrm{NiO}$, als Nickelo-nickelit aufzufassen ist. Tatsächlich sind solche Nickelite auch mit anderen Basen $(\mathrm{K}, \mathrm{Na}, \mathrm{Ba})$ als wohl gekennzeichnete Verbindungen dargestellt, während andererseits Derivate von $\mathrm{Ni}_{2} \mathrm{O}_{3}$, die mit Sicherheit auf dreiwertiges Nickel zurückgeführt werden können, bisher nicht erbalten worden sind. Schon vor einer Reihe von Jahren haben wir mit Versuchen zur Darstellung solcher Verbindungen begonnen, ${ }^{3}$ um damit zu einem einwandfreien Nachweis der Existenz dreiwertigen Nickels zu gelangen, doch sind alle Bemühungen in dieser Richtung bis heute ohne sicheren Erfolg geblieben. Wohl ist es gelungen, auf verschiedenen Wegen eine Reihe von teils ziemlich beständigen, meist sehr schön dunkelrot gefärbten Lösungen darzustellen, die man versucht sein könnte, für Lösungen von Nickelisalzen zu halten; eine genauere Untersuchung ergab jedoch in fast allen Fällen, dals man es hier nur mit kolloidalen Lösungen von Nickelsuperoxyd zu tun hat, zu deren Bildung das Superoxyd, namentlich bei Gegenwart schwacher Säuren, eine ausgesprochene Neigung besitzt.

'Atti dei Line. 14 II. (1905), 234; Garin. chim. ital. 36 I. (1906), 58.

$2 \mathrm{Vgl}$. besonders Förster, Z. f. Elektrochem. 13 (1907), 414; K. A. HoF. mann u. E. Seller, Ber. Dtsch. Chem. Ges, 39 (1906), 3187.

3 Tusaxd, Z. anorg. Chem. 45 (1905), 366 . 
Auch die vereinzelt in der Literatur sich vorfindenden Angaben über Erscheinungen, aus denen die Beobachter auf die Existenz von Verbindungen dreiwertigen Nickels schlielsen, haben wir einer eingehenden Nachprüfung unterzogen, konnten aber nur finden, dafs jene Schlufsfolgerungen auf Irrtümern beruhen.

Wenn nur aber auch unsere Untersuchungen in der angegebenen Richtung im wesentlichen alle zu einem negativen Ergebnis führten, konnten wir dabei doch eine Reihe interessanter Beobachtungen machen, die einige irrtümliche Auffassungen über das Nickelsuperoxyd korrigieren und dazu beitragen werden, dasselbe durch eine Anzahl neuer Reaktionen näher zu charakterisieren.

\section{Einwirknng chemischer 0xydationsmittel auf Nickelverbindungen.}

Bei der Einwirkung chemischer Oxydationsmittel auf Nickeloverbindungen entstehen, so weit eine solche Einwirkung bei Abwesenbeit von freiem Alkali überhaupt stattfindet, stets nur schwarze Niederschläge von Nickelsuperoxyd. N. Ch. NÂG ${ }^{1}$ gibt allerdings an, beim Versetzen ron Nickelkarbonat mit Natriumacetat und Brom eine dem Kaliumbichromat ähnlich gefärbte Nickellösung erhalten zu haben, doch ist uns diese Reaktion unter den verschiedensten Bedingungen niemals geglïckt; wir kommen weiter unten darauf zurück. Nur in einem Falle, als wir nämlich auf konzentrierte, mit Nickelsalz versetzte und auf $0^{0}$ abgekühlte Lösungen von Kaliumbikarbonat Ozon (ca. $10 \% \mathrm{ig}$ ) einwirken liefsen, erhielten wir ziemlich rasch eine schön tiefrot gefärbte, anfänglich klar durchsichtige Lösung, aus der sich erst nach längerer Zeit Nickelsuperoxyd ausschied. Bei höherer Temperatur tritt diese Ausscheidung viel früher ein, so dafs die Lösungen meist von vornherein trüb erscheinen. Diese rote Lösung ist identisch mit der schon früher auf elektrolytischem Wege ${ }^{2}$ erhaltenen, die damals als Lösung eines komplexen Nickelikarbonats angesprochen wurde. Eine genauere Untersuchung der Lösung ist infolge ihrer aufserordentlichen Zersetzlichkeit nicht möglich; es scheint uns jedoch heute gewifs, ${ }^{3}$ dafs hier nicht ein Nickelisalz, sondern eine unbeständige kolloidale Lösung von Nickelsuperoxyd vorliegt, das nach einiger Zeit teils von der Kohlensäure unter Reduktion gelöst, ${ }^{4}$ teils präzipitiert wird.

$1 Z$. anarg. Chem. 13 (1897), 16.

2 Tuband, $Z$. anorg. Chem. 45 (1905), 366; vgl. auch weiter unten.

${ }^{3}$ Siehe weiter unten.

${ }^{4}$ Kohlensäure und auch Bikarbonatlösungen lösen sehr fein verteiltes 
Andere einfache und komplexe Nickelsalze werden von Ozon nur sehr langsam angegriffen ${ }^{1}$ unter Ausscheidung geringer Nengen von Superoxyd, in keinem Falle konnten wir eine ähnliche Erscheinung beobachten wie bei den Bikarbonatlösungen.

Auch gewöhnlicher Sauerstoff kann oxydierend wirken auf Nickelverbindungen; besonders ist seit langem bekannt, dals feuchtes Nickelhydroxydul an der Luft die Fähigkeit erlangt, Jod in Freiheit zu setzen, und dals es, etwas alkalihaltig, bei Gegenwart von schwefliger Säure bzw. Sulfit sehr rasch in das schwarze Superoxyd übergeht. Wie wir vor kurzem mitteilten, ${ }^{2}$ lälst sich eine solche autoxydierende Wirkung auch bei der Nickelcyanwasserstoffsäure nachweisen, doch tritt dabei als Produkt des Autoxydationsworganges ausschliefslich das Wasserstoffsuperoxyd in die Erscheinung.

\section{Umsetzung von Nickelsuperoxyd mit Säuren.}

Ein zweiter Weg, zu Verbindungen dreiwertigen Nickels zu gelangen, schien in der Umsetzung von Nickelsuperoxyd mit Säuren gegeben. Zwar löst sich unter gewöhnlichen Verhältnissen das Nickelsuperoxyd in den meisten Säuren sehr rasch unter gleichzeitiger Reduktion, doch lassen sich die Bedingungen hier leicht so treffen, dals die Reduktion ziemlich langsam verläuft. Solche Versuche sind auch bereits von BENEDiCT ${ }^{3}$ angestellt worden, und er nimmt auch mit Bestimmtheit an, auf diesem Wege wirklich Nickelisalze, besonders Nickeliacetat und Nickelisulfat, letzteres sogar in fester Form, erhalten zu haben. Wir werden uns deshalb mit diesen Versuchen hier besonders zu beschäftigen haben.

BENEDICr behandelt frisch gefälltes, erst mit heilsem, dann mit Eiswasser sorgfältig ausgewaschenes Nickelsuperoxyd direkt auf dem Filter mit $90 \%$ iger, auf $0^{\circ}$ abgekühlter Essigsäure. Man erhält so ein kohlschwarzes Filtrat, das sich in der Kälte nach einiger Zeit, beim Erwärmen momentan unter Bildung von Nickeloacetat zersetzt. Alkalilauge fällt schwarzes Superoxyd; Reduktionsmittel bewirken sofortigen Farbumschlag in Grün, aus Jodkalium wird Jod ausgeschieden, während Bromkalium nicht merklich zersetzt wird.

Nickelsuperoxyd ziemlich rasch; gröbere Teilchen werden dagegen nur langsam angegriffen.

1 Vgl. auch MajJfert, Compt. rend. 94 (1882), 860.

2'Tubandt u. Riedel, Ber. deutsch, chem. Ges. 44, Heft 13.

${ }^{3}$ Journ. Amer. Chem. Soo. 28 (1906), 171.

Z. snorg. Chern. Bd. 72 . 
Wir können die auf diese Beobachtungen sich stützende Ansicht Benedicts, dals die erwähnte schwarze Lösung Nickeliacetat enthält, nicht teilen; es handelt sich, wie auch die Untersuchung der Lösung im Ultramikroskop zeigte, um nichts anderes als eine Suspension verhältnismälsig grober Teilchen von Nickelsuperoxyd. Dementsprechend kann man auch zuweilen deutlich beobachten, dals der allmählich sich vollziehende Farbumschlag der Flüssigkeit im Reagenzglas oben beginnt und nach unten fortschreitet, weil eben das Superoxyd während des Redultions- und Lösungsvorganges nach unten sinkt. Weiter ist es für die Herstellung der schwarzen Flüssigkeit durchaus nicht nötig, Essigsäure so hoher Konzentration anzuwenden, $50 \%$ ige Säure oder auch eine mit Essigsäure versetzte Lösung von Natriumacetat führt zu genau denselben Erscheinungen. Besonderes Gewicht legt Benedict darauf, dafs die Behandlung des Superoxyds auf dem Filter geschieht, im Reagenzrohr soll sofortige Reduktion eintreten. Auch das ist nicht zutreffend; es bereitet gar keine Schwierigkeit, die Lösungen durch Übergielsen des Superoxyds mit Essigsäure im Reagierrohr zu bereiten. Diese sind indes meist nicht wie die auf dem Filter dargestellten schwarz, sondern schön rotbraun gefärbt. Sie lassen sich kalt ohne Rückstand filtrieren und geben bei Verdünnen mit Essigsäure oder ebensogut mit Wasser klar durchsichtige bräunlich rote Lösungen. Auch diese roten Lösungen enthalten nach den Untersuchungen im Ultramikroskop kein Nickeliacetat, sondern Nickelsuperoxyd in kolloidähnlichem Zustande. Wahrscheinlich hat auch NÂG solche kolloidale Lösungen in Händen gehabt, als er Nickelkarbonat mit Natriumacetat und Brom bebandelte. ${ }^{1}$ Die Bildung solcher Lösungen ist übrigens nicht nur auf die Essigsäure beschränkt, auch andere schwache Säuren, wie Weinsäure, Zitronensäure u. a. sind dazu befähigt. Nicht selten kann man auch schon beim Auswaschen gröfserer Mengen von Superoxyd mit kaltem Wasser beobachten, dals, nachdem der Vorgang eine Zeitlang ganz glatt vonstatten gegangen, plötzlich ein schwarzes oder rotviolettes Filtrat durch das Filter geht, das in den meisten Fällen das Superoxyd ziemlich bald ausscheidet, zuweilen jedoch auch tagelang unverändert haltbar ist, und natürlich auch alle Reaktionen gibt, die BENEDrct für die essigsaure Lösung angibt. Recht haltbare, besonders schön rot gefärbte und vollkommen klare Lösungen erhielten wir auch mit verdünnter Phosphorsäure ${ }^{2}$

1 Siehe oben.

2 Wir haben Lösungen von Ortho- und Metaphosphorsäure verwendet, beide mit gleichem Ergebnis. 
die rote Farbe einer mit etwa 5\% iger Metaphosphorsäure (aus $\mathrm{P}_{2} \mathrm{O}_{5}$ und Wasser dargestellt) bereiteten Lösung blieb in der Kälte fast einen ganzen Tag lang bestehen; sie liels sich mit Wasser beliebig verdünnen ohne jede Ausscheidung. Es erschien hier besonders verführerisch, an die Existenz eines Nickelisalzes zu glauben, nach dem Befund im Ultramikroskop lag indes auch hier nichts anderes als kolloidales Superoxyd vor.

In konzentrierten Lösungen von Phosphorsäure ist nichts von einer Bildung solcher kolloidaler Lösungen zu erkennen, wohl aber besitzen die entstehenden grünen Lösungen, nachdem alles Superoxyd verschwunden ist, eine sehr starke Oxydationswirkung. Aus Jodkalium wird auch bei grölster Verdünnung sofort Jod ausgeschieden, ebenso werden aus Brom- und Chlorkalium die Halogene in Freiheit gesetzt. Halbständiges Erwärmen im Wasserbade oder kurzes Aufkochen der Lösungen zerstört ihre oxydierende Wirkung nicht, bei gewöhnlicher Temperatur besalsen sie dieselbe auch nach wochenlangem Stehen noch. Wasserstoffsuperoxyd war in keinem Falle, weder in den frisch bereiteten Lösungen noch nach längerem Stehen derselben nachzuweisen. Dals in der Lösung keine Verbindung eines höherwertigen Nickels vorhanden war, wurde besonders noch dadurch dargetan, dal's nach dem Ausfällen des Nickels durch einen geringen Überschuis von Kalilauge das wieder angesäuerte Filtrat dieselbe Oxydationswirkung zeigte wie vorher.

Es kann also kaum ein Zweifel darüber bestehen, dafs man es hier mit der kürzlich von Schmiduin und MassinI ${ }^{1}$ durch Umsetzung von $\mathrm{P}_{2} \mathrm{O}_{5}$ bzw. Meta- oder Pyrophosphorsäure mit Wasserstoffsuperoxyd dargestellten, der Cakoschen Säure entsprechenden Phosphormonopersäure zu tun hat. Die Lösung zeigte auch die anderen be-

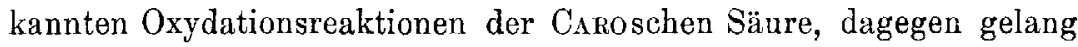
es uns nicht, mit Sicherheit die Oxydation von Mangansalzen zu Permangansäure festzustellen, welche nach Schmidurs und Massins die Phosphormonopersäure im Gegensatz zur Caroschen Säure zu bewirken imstande ist. Der Grund dafür liegt wahrscheinlich darin, dals in den von uns erhaltenen Lösungen die Persäure in zu geringer Konzentration vorhanden ist.

In starken Säuren, in denen das Nickelsuperoxyd sich sehr rasch auflöst, findet eine Bildung solcher kolloidaler Lösungen natürlich nicht statt, wenigstens nicht in wässerigen Lösungen ${ }^{2}$ der-

1 Ber. deutsch. ehem. Ges. $43(1910), 1162$.

2 Bezïgl. alkoholischer Lösungen vgl. S. 229. 
selben. Für die auffallenden Erscheinungen, die BEnEdict ${ }^{1}$ bei der Behandlung von Nickelsuperoxyd mit konzentrierten Lösungen von Kaliumhydrosulfat beobachtete, kommt also eine Deutung durch die Bildung solcher Lösungen nicht in Frage. Nickelisulfat hat BENEDiCT nach unseren Versuchen indes sicher auch hierbei nicht in Händen gehabt. Er beobachtete folgendes:

Übergielst man frisch gefälltes, wie oben behandeltes Nickelsuperoxyd auf dem Filter mit nicht zu stark gekühlter, nicht ganz gesättigter Kaliumhydrosulfatlösung, so erhält man zunächst ein intensiv grünes Filtrat, die späteren Anteile desselben sind schwächer grün gefärbt, dann so gut wie farblos und schliefslich rosa gefärbt. Diese Farbe vertieft sich allmählich zu einem ausgesprochenen Rot und wird in dem Mafse, als sich aus der Lösung beim Stehen grüne Kristalle ausscheiden, immer intensiver. Sowohl der farblose, als auch der rot gefärbte Anteil des Filtrates enthält reichliche Mengen von Nickel und besitzt eine sehr starke Oxydationswirkung, indem Jod und Brom augenblicklich, Chlor langsamer aus ibren Salzen ausgeschieden werden unter gleichzeitigem Umschlag der Farbe der Lösungen in Grün. In der Kälte bleibt die rote Farbe der Lösungen tagelang bestehen, Reduktionsmittel führen sofort Farbumschlag herbei. Die Lösung muls also, so schliefst Benedict aus diesen Erscheinungen, ein Salz des dreiwertigen Nickels enthalten. Dafs Natronlauge grünes Nickelhydroxydul und nicht das schwarze Sesquioxyd ausscheidet, führt er auf die bei der Umsetzung des Bisulfats mit $\mathrm{NaOH}$ auftretende hohe Reaktionswärme zurück. Nach ein bis zwei Tagen, nachdem die Ausscheidung der grünen Kristalle aus den roten Lösungen praktisch beendet ist, scheidet sich ein ziegelrotes amorphes Pulver aus, während die Lösung sich langsam entfärbt. Dieses Pulver, Nickelsulfat oder der Alaun desselben, ist unlöslich in Wasser, während es in schwefeliger Säure leicht mit grüner Farbe in Lösung geht.

Das sind zum Teil recht merkwürdige Eigenschaften, die nach diesen Beobachtungen BENEDIOTs das Nickelisulfat besitzen mülste. Besonders auffallend muls die gegen alles Erwarten grofse Beständigkeit des vermeintlichen Nickelisalzes erscheinen, welche die des entsprechenden Kobaltisulfates, das auf anderem Wege nicht eben schwierig $z \mathfrak{u}$ erhalten ist, bei weitem übertreffen würde, und die Möglichkeit, die Verbindung in gröfseren Mengen darzustellen und zu analysieren, recht aussichtsvoll erscheinen lassen müfste.

Unsere Versuche überzeugten uns jedoch bald, dal's hier zweifel-

${ }^{1}$ Journ. Amer. Chem. Soc. 28 (1906), 171. 
los ein Irrtum vorliegt. Eine grofse Anzahl von Versuchen, die mit aus reinstem Nickelosalz dargestelltem Nickelsuperoxyd ausgeführt wurden, lieferten bis zum Ende der Reaktion immer nur grüne Filtrate, je nach der Konzentration und Temperatur der angewandten Hydrosulfatlösung dunkler oder heller gefärbt, niemals aber konnten wir die Entstehung einer rötlich gefärbten Lösung beobachten, auch nicht, als wir die Fällung des Nickelsuperoxyds unter mannigfach verschiedenen äulseren Bedingungen, die auf die $\mathrm{Zu}$ sammensetzung der schwarzen Niederschläge ja bekanntlich von grofsem Einfluls sind, vornahmen.

Wohì aber besalsen die grünen Lösungen alle eine kräftige Oxydationswirkung und behielten diese auch noch nach dem Erwärmen im Wasserbad und wochenlangem Stehen bei Zimmertemperatur nahezu unverändert bei. Eine Nickeliverbindung, deren Färbung etwa durch das grüne im Überschuls vorhandene Nickelosalz verdeckt sein konnte, lag also sicher nicht vor; das wurde im besonderen noch durch die quantitative Bestimmung des Oxydationswertes und des Nickelgehaltes der Lösung (nach dem Auskristallisieren der Hauptmenge des Nickelosalzes) bestätigt: die Menge des titrierbaren Sauerstoffes war mehr als dreimal so grols, als alles vorhandene Nickel in dreiwertiger Form hätte enthalten können, und schliefslich fand sich der Oxydationswert der Lösung auch fast unverändert wieder, als alles Nickel mit Kalilauge ausgefällt worden war.

Für die starke Oxydationswirkung der Lösung konnten also nur in Betracht kommen: Perschwefelsäure, CArosche Säure und vielleicht Wasserstoffsuperoxyd. Es ist uns jedoch niemals gelungen, Wasserstoffsuperoxyd nachzuweisen, obwohl wir auf diese Versuche mit Rücksicht auf die Angaben Tanatars, ${ }^{1}$ wonach Nickelsuperoxyd als einziges „unechtes“ Superoxyd bei der Umsetzung mit verdünnter Schwefelsäure Wasserstoffsuperoxyd bilden soll, ganz besonders unsere Aufmerksamkeit richteten, auch nicht, als wir statt Kaliumbydrosulfat Schwefelsäure verschiedener Konzentration verwendeten, und dabei auch genau die von TANatar angegebenen Bedingungen einhielten. Wir haben darüber vor kurzem bereits an anderer Stelle berichtet. ${ }^{2}$ Dagegen zeigen die Lösungen von Nickelsuperoxyd in Schwefelsäure oder konzentrierter Kaliumhydrosulfatlösung alle die bekannten Realtionen der CARoschen Säure,

1 Ber. Dtsch. Chem. Ges. 33 (1900), 205; 12 (1909), 1516.

- Ber. Dtsch. Chem. Ges.44, Heft 13. 
während es wieder nicht gelang, Perschwefelsäure neben der $\mathrm{C}_{A}$ Roschen Säure mit Sicherheit nachzuweisen.

Alle von BENEDICT beobachteten Reaktionen seiner "Nickelisalz"lösung sind nichts anderes als Reaktionen der CARoschen Säure, nur die Rotfärbung des Endfiltrates und die Ausscheidung des ziegelroten Pulvers, die von ihm beobachtet wurden, bleiben zu erklären. Wir glauben, dafs uns auch diese Erklärung schliefslich gelungen ist.

I) a wir die von ihm geschilderten Erscheinungen, wie oben erwähnt, in einer langen Reihe von Versuchen niemals erhalten hatten, befestigte sich die Vermutung, dafs es sich um eine Verunreinigung des verwendeten Nickelsalzes handeln könnte, und zwar, wie es am nächsten liegt, um eine solche durch Kobalt oder Mangan. Kobaltihydroxyd ist in Kaliumhydrosulfatlösung sehr viel schwerer löslich als Nickelperoxyd, so dals aus einem Gemisch beider sich zuerst hauptsächlich das letztere lösen, die Hauptmenge des Kobalts sich also im Endfiltrat finden wird, und dort vielleicht die beobachtete Rotfärbung der Lösung bedingen konnte. Zusatz kleiner Mengen von Kobalt, wie sie etwa in den gewöhnlichen Handelspräparaten von Nickelsalzen vorkommen, zu der reinen Nickelsalzlösung vor der Superoxydfällung änderte jedoch niclits Wesentliches an den mit kobaltfreien Lösungen beobachteten Erscheinungen. Nun hatten wir weiter gefunden, dals Nickelsuperoxyd die Fähigkeit besitzt, Manganverbindungen in schwefelsaurer Lösung zu Permangansäure zu oxydieren. Als wir nun unserem reinen Nickelsalz ror der Fällung des Superoxyds eine Spur von Manganosalz hinzufügten und das Superoxyd dann auf dem Filter mit Kaliumhydrosulfat bebandelten, beobachteten wir, dals das anfünglich stark grüne Filtrat in den weiteren Fraktionen allmählich verblalste, farblos und schliefslich rot wurde, dafs die Rotfärbung in dem Malse, als aus der Lösung das sehr schwer lösliche Nickelkaliumsulfat auskristallisierte, sich vertiefte, dafs aus der roten Flüssigkeit sich schliefslich ein ziegelroter pulveriger Niederschlag ausschied, kurz, genau alle die Erscheinungen, die Benedict anführt. Lösung und Niederschlag, die natürich starke Oxydationswirkung zeigten und sich in allem genau verhielten wie BENEDICr beschreibt, enthielten neben reichlichen Mengen von Nickel deutlich nachweisbare Mengen yon Mangan, je nach der ursprünglich hinzugefügten Manganmenge als Permangansäure oder Manganisulfat. Es genügen zur Hervorrufung der beschriebenen Erscheinungen so geringe Mengen von Mangan, dafs man sie direkt als eine recht brauchbare Methode zum Nach- 
weis kleinster Spuren von Mangan neben sehr viel Nickel verwenden kann. Gemindert wird die Empfindlichkeit der Reaktion nur dadurch, dafs das Auftreten jener Erscheinungen an eine ziemlich eng begrenzte Konzentration der Kaliumhydrosulfatlösung gebunden ist, da bei vollkommen gesättigter Lösung auf dem Filter sich bereits grofse Mengen von Nickelkaliumsulfat ausscheiden und verhindern, dals alles Superoxyd in Reaktion tritt, bei mälsigem Verdünnen der gesättigten Lösungen aber das Nickel in so grolsen Mengen in Lösung bleibt, dals die intensiv grüne Farbe derseiben das Permanganat vollkommen verdeckt, falls Mangan wirklich nur spurenweise vorhanden ist. An Stelle oder neben dem pulverigen Niederschlag erhält man zuweilen auch schön rot gefärbte Kristalle, die neben Nickel- und Kaliumsulfat winzige, doch deutlich nachweisbare Spuren von Manganisulfat enthalten, dem sie ihre Rotfärbung verdanken. Schlielslich haben wir das rote Filtrat und den daraus sich ausscheidenden festen roten Körper auch bei einigen Nickelsalzpräparaten, darunter auch einem als rein bezogenen, ohne besonderen Manganzusatz erhalten, konnten aber in allen Fällen nachweisen, dafs es sich auch hier immer um Mangan handelte, das in den betreffenden Präparaten als Verunreinigung enthalten war.

Wir zweifeln nach alledem nicht daran, dafs Nickelisulfat bei der beschriebenen Behandlung von Nickelsuperoxyd mit Kaliumhydrosulfatlösung nicht entsteht, und dafs auch BENEDICT kein solches, sondern eine Manganverbindung vor sich gehabt bat. Letzteres glauben wir um so mehr annehmen zu dürfen, als er die vor 6 Jahren in Aussicht gestellte Analyse des roten Niederschlags, den er als Nickelisulfat bzw. dessen Alaun ansprach, unseres Wissens bisher schuldig geblieben ist.

Nickelperoxyd besitzt also die Fähigkeit in Gegenwart von Schwefelsäure niedere Oxyde des Mangans zu Permangansäure zu oxydieren, eine Fühigkeit, die von den Superoxyden, abgesehen von Perschwefelsäure und Phosphormonopersäure, nur noch dem Bleisuperoxyd in Gegenwart von Salpetersäure zukommt. Man kann das Nickelsuperoxyd also wie Bleisuperoxyd als Reagens auf Mangan verwenden. Am besten versetzt man die manganhaltige Lösung mit Nickelsuperoxyd und dann mit einigen Tropfen konzentrierter Schwefelsäure, das Mangan gibt sich dann augenblicklich an einer intensiven Rotfärbung der Lösung zu erkennen. Es ist nicht nötig, frisch gefülltes Nickelsuperoxyd zu verwenden, getrocknetes Oxyd wirkt ebenso gut, wenn nicht besser, da es sich langsamer löst. Da Nickelsuperoxyd in trockenem Zustande sehr gut haltbar 
ist, ${ }^{1}$ lälst die Reaktion an Einfachheit nichts zu wünschen übrig. Kleine Spuren von Permangansäure werden allerdings leicht durch die grüne Nickelfarbe verdeckt, nimmt man jedoch das Spektroskop zu Hilfe, so ist die Permangansäure auch in solchen Lösungen meist noch deutlich an den charakteristischen Absorptionsstreiten zu erkennen. Die Reaktion verläuft auch in der Kälte (Zusatz verdünnter Schwefelsäure), nur ein wenig langsamer. Das bringt den Vorteil, dals Mangan auch bei Gegenwart von Chlorwasserstoffsäure, und zwar verhältnismälsig grofser Mengen, noch deutlich erkannt werden kann, da stark verdünnte Salzsäure sowohl mit der sich bildenden Permangansäure als auch mit dem in kompakten Stücken angewandten Nickelsuperoxyd nur langsam reagiert, jedenfalls langsamer als die Permangansäure in der schwefelsauren Lösung erzeugt wird. Allerdings sind hierbei nicht zu kleine Mengen von Mangan vorausgesetzt, Spuren können in Gegenwart von Halogen nicht mehr erkannt werden.

Ist Mangan in der schwefelsauren Lösung in gröfseren Mengen vorhanden, so wird es durch Nickelsuperoxyd nicht zu Permanganat, sondern zu Manganisulfat oxydiert und kann als solches erkannt werden; durch Bleisuperoxyd und Salpetersäure werden manganreiche Lösungen dagegen nicht erkennbar oxydiert, weil das Manganinitrat, ${ }^{2}$ das bei der Umsetzung von Permangansäure mit überschüssigem Manganosalz entsteht, äufserst unbeständig ist, während das Manganisulfateine verhältnismäisig viel grölsere Beständigkeit besitzt.

Mit wenig Mangan gibt Nickelsuperoxyd natürlich auch in salpetersaurer Lösung die Permanganatreaktion, es bedarf hierbei nur eines ganz schwachen Erwärmens der Lösung, um sie augenblicklich hervorzurufen; selbstverständilich verhindern gröfsere Manganmengen auch hier wie bei der Reaktion mit Bleisuperoxyd ihr Hervortreten, doch kann die Gegenwart von Mangan durch Nickelsuperoxyd auch dann noch an der Ausscheidung von Braunstein erkannt werden.

Auch in phosphorsaurer Lösung wird Manganosalz leicht durch Nickelsuperoxyd zu Permangansäure bzw. Manganiphosphat oxydiert, doch ist die Reaktion weniger empfindlich als in schwefelsaurer und salpetersaurer Lösung.

Verwendet man an Stelle wässeriger Lösungen der Säuren solche in absolutem Alkohol und kühlt diese mit fester Kohlensäure stark ab, so erhält man mit Nickelsuperoxyd, das erst mit Wasser,

${ }^{1}$ Vgl. auch Fönster, Zeitschr. f. Elektroehem. 13 (1907), 418.

"Über die Bildung der Nitrate von drei- und vierwertigem Mangan werden wir demnächst berichten. 
dann mit Alkohol gewaschen wurde, auch mit starken Säuren, wie Schwefelsäure oder Chlorwasserstoff, einige Zeit beständige, tiefrote Lösungen, die sich gut filtrieren lassen. Auch in diesem Falle handelt es sich indes aller Wahrscheinlichkeit nach um nichts anderes als kolloidale Lösungen von Nickelsuperoxyd, wenigstens erwies sich die Lösung in alkoholischer Schwefelsäure nach dem Erwärmen auf $0^{\circ}$, bei welcher Temperatur sie sich einige Zeit noch klar durchsichtig erhielt, im Ultramikroskop als solche. Bei gewöhnlicher Temperatur verschwindet das Superoxyd rasch unter Bildung ron Nickelosulfat und Oxydation des Alkohols zu Aldehyd.

\section{Elektrolytische Oxydation von Nickeloverbindungen. ${ }^{1}$}

Bei den sehr zahlreichen Versuchen, die wir unternahmen, durch anodische Oxydation einfacher und komplexer Nickelsalze zu Verbindungen dreiwertigen Nickels zu gelangen, konnten wir, wie schon in einer früheren Mitteilung ${ }^{2}$ berichtet wurde, nur bei konzentrierten Kaliumbikarbonatlösungen Erscheinungen beobachten, die auf die Bildung eines Nickelisalzes hinzudeuten schienen. Auch bei der Elektrolyse cyankalischer mit etwas freiem Alkali rersetzter Nickellösungen zwischen Platinelektroden färbt sich die Flüssigkeit zwar gewöhnlich vorübergehend tiefrot, und man hat die Vermutung ausgesprochen, es hier mit einem Nickelisaiz zu tun zu haben. Man kann sich indes namentlich bei Anwendung eines Diaphragmas leicht überzeugen, dafs nur die Kathodenflüssigkeit die intensive Rotfärbung annimmt, während an der Anode keine Veränderung sichtbar wird. Die rote Lösung reduziert energisch Silber-, Blei-, Wismut-, Quecksilber-, Arsenverbindungen zu den betreffenden Metallen, und wird durch Oxydationsmittel augenblicklich entfärbt. Es kann also kein Zweifel bestehen, dafs es sich hier nicht um eine Verbindung höherwertigen Nickels, sondern des Suboxyds $\mathrm{Ni}_{3} \mathrm{O}$ handelt, die nach TH. Moore ${ }^{3}$ auch auf rein chemischen Wege, durch Einwirkung von Zink, Natriumamalgam, Zinnchlorür usw. auf cyankalische Nickellösungen erhalten werden kann.

Elektrolysiert man mit einem Nickelsalz versetzte, auf $0^{\circ}$ abgekühlte konzentrierte Lösungen von Kaliumbikarbonat zwischen Platinelektroden (man wendet am besten Drahtnetz an), so verbreiten sich alsbald unter gleichzeitiger Sanerstoffentwickelung von

${ }^{1}$ Diese Versuche wurden ausgeführt von $O$. Frrtze, bezüglich der Einzelheiten vgl. dessen Inaug.-Diss., Halle 1909.

2 Tobandt, $Z$. anorg. Chem, 45 (1905), 366.

${ }^{3}$ Chem. Nenes 68 (1894), 295; 71 (1895), 81. 
der Anode aus rote Schlieren in die Flüssigkeit, die an der Kathode unter Abscheidung von Nickel rasch wieder verschwinden. Führt man ein Diaphragma ein, so ist nach wenigen Minuten die ganze Anodenflüssigkeit schön tiefrot gefärbt. Allmählich wird die Lösung fast schwarz; in dünnen Schichten oder nach dem Verdünnen mit kalter Kaliumbikarbonatlösung oder mit Eiswasser zeigt sie jedoch noch immer die schöne rote Farbe, ist vollkommen klar und ohne Rückstand filtrierbar. Die Bildung der roten Lösungen tritt nur bei niederen Stromdichten ein, die obere Grenze liegt bei etwa 1 Ampere; bei höheren Stromstärken wird nur noch etwas Superoxyd auf der Anode abgeschieden. Auch bei niederer Stromdichte findet häufig nicht sofort Oxydation der Nickellösung statt; durch kurze kathodische Polarisation, besser noch durch mehrmaligen raschen Wechsel der Stromrichtung kann man die Reaktion an der Nickelanode indes immer leicht einleiten. Bei Stromdichten bis höehstens 0.4 Ampere und einem Nickelgehalt ${ }^{1}$ der Lösungen bis etwa $0.015 \mathrm{~g}$ in $100 \mathrm{ccm}$ liees sich bei Anwendung grol'ser Nickeldrahtnetzelektroden scheinbar alles gelöste Nickel in den dreiwertigen Zustand überführen, ${ }^{2}$ bei höheren Stromdichten oder bei Anwendung von Platinelektroden war der Oxydationswert der Lösungen jedoch stets erheblich geringer. Oft treten bei der Elektrolyse am Amperemeter und Voltmeter starke periodische Schwankungen auf, ähnlich denen, wie sie von T'HIes und Windelschmidt ${ }^{3}$ bei ammoniakalischen und oxalsauren Nickellösungen beobachtet worden sind, und die, wie dort, durch die abwechselnde Bildung und Wiederauflösung einer Superoxydschicht auf der Anode hervorgerufen werden. Mannigfache Versuche konzentriertere Lösungen zu gewinnen führten alle zur Ausscheidung von Nickelsuperoxyd, lange

1 Nickelkarbonat ist in der gesättigten Kaliumbikarbonatlösung nur in sehr geringer Menge löslich (es bildet sich das sehr wenig lösliche Doppelsalz $\left.\mathrm{NiCO}_{3} \cdot \mathrm{KHCO}_{3}, 4 \mathrm{H}_{2} \mathrm{O}\right)-100 \mathrm{cem}$ halten bei $0^{\circ}$ nur etwa $0.0009 \mathrm{~g} \mathrm{Ni}$ gelöst; doch lassen sich leicht wenigstens kurze Zeit haltbare Lösungen bis zu einem Nickelgebalt von etwa $0.1 \mathrm{~g}$ in $100 \mathrm{ccm}$ darstellen.

2 Die Bestimmung des Nickelgehaltes der Lösung geschicht am beston durch Elektrolyse der mit konz. Ammoniak (ea. $30 \mathrm{cem}$ Ammoniak auf $100 \mathrm{ccm}$ der Lösung) versetzten Bikarbonatlösung; das Nickel scheidet sich daraus rasch schön metallglïnzend $\mathbf{a b}$, ein Kohlenstoffgehalt des niedergeschlagenen Nickels konnte niemals konstatiert werden. - Der Oxydationswert der Lösungen wurde durch Zusatz arseniger Säure und Rücktitration derselben mittels Jodlösung, oder auch durch vorsichtiges Einfliefsenlassen der roten Lösung in angesäuerte Jodkaliumlösung und Titration des ansgeschiedenen Jods mittels Thiosulfat ermittelt.

3 Zeitschr. f. Elektrohem. 13 (1907), 317. 
ehe alles Nickelsalz oxydiert war. Wesentliche Verminderung der Konzentration der Kaliumbikarbonatlösung oder Erhöhung der Temperatur über $0^{\circ}$ beeinträchtigt die Bildung der roten Lösungen in hohem Malse. Aber auch die kalten Lösungen sind aufserordentlich leicht zersetzlich, höchstens 3 Stdn. nach Stromunterbrechung ist die rote Farbe der Lösung wieder in Grün übergegangen, bei höherem Nickelgehalt der Lösung scheidet sich auch etwas Nickelsuperoxyd aus. Setzt man die Elektrolyse fort, verschwindet die rote Farbe der Lösungen ebenfalls wieder, nur wird in diesem Falle fast alles Nickel an Tonzelle und Gefälswandung als Superoxyd ausgeschieden.

Die roten Lösungen besitzen ein sehr starkes Oxydationsvermögen; durch Reduktionsmittel oder schwaches Erwärmen werden sie momentan entfärbt. Verdünnen mit Kiswasser führt dagegen keine schnellere Zersetzung herbei. Starke Laugen fällen schwarzes Oxyd.

Dieselben Lösungen, allerdings mit noch geringerem Nickelgehalt, kann man auch durch anodische Auflösung von Nickelelektroden in reiner konzentrierter Kaliumbikarbonatlösung erhalten. Bei niederen Stromdichten geht, wie ein angeschaitetes Voltmeter zeigte, die Nickelanode in der Bikarbonatlösung zunächst mit niederer allmählich nur ganz wenig steigender Spannung in Lösung, bis dann auf einmal ein plötzlicher starker Spannungsanstieg erfolgt. Dieser tritt um so früher ein, je höher die Stromdichte, je niedriger die Konzentration der Bikarbonatlösung ist; Erböhung der Temperatur und starke Rührung des Elektrolyten verzögern den Eintritt der plötzlichen Spannungsänderung. Solange die niedere Spannung anhält, geht das Nickel quantitativ als Ni"-Ion in Lösung; die Anode bleibt zunächst vollkommen blank, später überzieht sie sich mit einer dünnen grüngrauen Schicht von Nickelkarbonat. Mit Eintritt der hohen Spannung stellt sich sofort Gasentwickelung ein, während der Elektrolyt rasch rote Farbe annimmt. Auch bei Stromdichten, die fast sofort nach Stromschlufs die höhere Spannung herbeiführen, kann man gleich am Anfang deutlich die Entstehung roter Schlieren an der Anode beobachten. Unterbricht man den Strom, nachdem die hohe Spannung erreicht ist, und lälst die stromlose Elektrode längere Zeit mit der Bikarbonatlösung in Berührung, so zeigt sie nach Anlegung niederer Stromdichten wieder die niedere Spannung. Nach Eintritt der hohen Spannung ist die Auflösung der Nickelanode nur noch gering, erscheint jedoch nie ganz aufgehoben. Bei Stromdichten von 0.1 bis 0.4 Ampere, die sich auch sonst für die 
Bildung der roten Lösung am günstigsten erwiesen, erhält man in kurzer Zeit eine tief braunrot gefärbte Lösung, obwohl bei diesen Stromdichten bei niederer Temperatur sofort die hohe Spannung auftritt. Auch in reinem mit Kohlensäure gesättigtem Wasser wird die Nickelanode stark angegriffen. Die Nickelanode verhält sich in Bikarbonatlösung also ganz ähnlich wie die Eisen- ${ }^{1}$ und Kobaltanode. ${ }^{2}$ Beiläufig sei hier auch erwähnt, dafs in Alkalikarbonat16 sung, wenn man durch fortgesetzte Erneuerung des Elektrolyten die Bildung von Bikarbonat an der Anode möglichst verhindert, Nickel vollkommen unangreifbar erscheint; dagegen fanden wir die schon von RIESENFELD ${ }^{3}$ gemachte Beobachtung bestätigt, dafs Nickelanoden in starken Lösungen von reinem Kalium- oder Natriumhydroxyd merklich angegriffen werden; bei niederen Stromdichten kann man, namentlich bei böherer Temperatur, in konzentrierten Laugen nach $1 / 4$ bis $1 / 2$ Min. deutlich einen plötzlichen Spannungsanstieg beobachten, vor Eintritt desselben wird ausschlielslich Nickelohydroxyd gebildet, das teilweise in Lösung geht; erst mit der Spannungserhöhung tritt Sauerstoffentwickelung und Oxydation des Hydroxyduls zu Superoxyd ein.

Unsere frühere Ansicht, dås die roten Bikarbonatlösungen ein lromplexes Nickelisalz enthalten, möchten wir heute nach den inzwischen auch bei den anderen oben beschriebenen Lösungen gemachten Erfahrungen nicht mehr aufrecht erhalten, das ganze Verhalten der Lösungen deutet vielmehr darauf hin, dafs auch hier nur eine kolloidale Lösung von Nickelsuperoxyd vorliegt.

Alle Versuche, aus den verschiedenen roten Lösungen eine Verbindung höherwertigen Nickels zu isolieren, blieben dementsprechend völlig ergebnislos und müssen nach unseren Erfahrungen als aussichtslos betrachtet werden. Jedenfalls aber ist einstweilen kein Grund vorhanden, nach den bekannten Tatsachen die Existenz eines Nickelisalzes als erwiesen oder auch nur als wahrscheinlich zu betrachten.

${ }^{1}$ Haber und Goldschmid, Zeitsehr. f. Elektrochem. 12 (1906), 49.

2 Fritze, Diss., Halle 1909.

3 Zeitschr. f. Elektrochem. 12 (1906), 621.

Italle, Chemisches Institut der Universitüt.

Bei der Redaktion eingegangen am 19. August 1911. 